\title{
Recurrent CME-like Eruptions in Emerging Flux Regions. II. Scaling of Energy and Collision of Successive Eruptions
}

\author{
P. Syntelis ${ }^{1}$ (1), V. Archontis ${ }^{1}$, and K. Tsinganos ${ }^{2}$ (1) \\ ${ }^{1}$ St. Andrews University, Mathematics Institute, St. Andrews KY16 9SS, UK; ps84@st-andrews.ac.uk \\ ${ }^{2}$ Section of Astrophysics, Astronomy and Mechanics, Department of Physics, University of Athens, Panepistimiopolis, Zografos 15784, Athens, Greece \\ Received 2018 November 27; revised 2019 April 3; accepted 2019 April 5; published 2019 May 3
}

\begin{abstract}
We present results of three-dimensional MHD simulations of recurrent eruptions in emerging flux regions. The initial numerical setup is the same as that in the work by Syntelis et al. Here, we perform a parametric study on the magnetic field strength $\left(B_{0}\right)$ of the emerging field. The kinetic energy of the produced ejective eruptions in the emerging flux region ranges from $10^{26}$ to $10^{28} \mathrm{erg}$, reaching up to the energies of small coronal mass ejections. The kinetic and magnetic energies of the eruptions scale linearly in a logarithmic plot. We find that the eruptions are triggered earlier for higher $B_{0}$ and that $B_{0}$ is not directly correlated to the frequency of occurrence of the eruptions. Using large numerical domains, we show the initial stage of the partial merging of two colliding erupting fields. The partial merging occurs partly by the reconnection between the field lines of the following and the leading eruption at the interface between them. We also find that tether-cutting reconnection of the field lines of the leading eruption underneath the following eruption magnetically links the two eruptions. Shocks develop inside the leading eruption during the collision.
\end{abstract}

Key words: magnetohydrodynamics (MHD) - methods: numerical - Sun: activity - Sun: interior - Sun: magnetic fields

\section{Introduction}

Most of the activity in the Sun appears to be directly connected with the properties of the solar magnetic fields. Due to dynamo action, magnetic fields, e.g., in the form of flux tubes, are generated inside the convection zone and give rise to sunspots and active regions (ARs) when they emerge to the solar surface (Parker 1955). ARs are the sources of the most intense solar phenomena, such as flares and coronal mass ejections (CMEs). The emergence of magnetic flux is also associated with less energetic and smaller-scale events, such as small filament eruptions and microsigmoids (e.g., Raouafi et al. 2010). Most eruptive phenomena are commonly related to twisted magnetic flux tubes in the solar atmosphere called flux ropes (FRs). A number of observational studies identify the presence of FRs in ARs before an eruption and study their formation (e.g., Green \& Kliem 2009; Cheng et al. 2011; Green et al. 2011; Patsourakos et al. 2013), the pre-eruptive phase (e.g., Canou \& Amari 2010; Vourlidas et al. 2012; Syntelis et al. 2016), and the triggering of the eruptions (e.g., Zuccarello et al. 2014; Chintzoglou et al. 2015; Yardley et al. 2018). It is also common for a single AR to produce more than one eruption (recurrent eruptions, e.g., Nitta \& Hudson 2001; Wang et al. 2013). The erupting FRs have been also identified in coronagraphic observations (e.g., Vourlidas et al. 2013). Understanding the FRs formation processes and the triggering mechanisms is critical, because these phenomena affect the terrestrial space environment (e.g., Patsourakos et al. 2016).

A number of models of solar eruptions require the presence of an FR prior to the eruption (e.g., Török \& Kliem 2005; Mackay \& van Ballegooijen 2006; Manchester et al. 2008; Török et al. 2011). Other models demonstrate the formation of an FR in a highly sheared AR during the eruption (e.g., Antiochos et al. 1999; Lynch et al. 2008). An important parameter for the FR formation prior to the eruption is the presence of shearing and converging motions along and toward a polarity inversion line (PIL; e.g., Magara \& Longcope 2001; Archontis \& Török 2008; DeVore \& Antiochos 2008; Aulanier et al. 2010). Due to these motions, a strong current forms above the PIL. There, the field lines reconnect and start forming an FR (e.g., van Ballegooijen \& Martens 1989). Flux-emergence models have shown that shearing and convergence motions develop naturally during the partial emergence of a subphotospheric FR (e.g., Fan 2001; Manchester 2001; Manchester et al. 2004). The expansion of the emerging field above the solar surface is commonly associated with untwisting of the embedded field lines and rotation of the emerging bipolar pair of sunspots (Fan 2009; Sturrock et al. 2015; Sturrock \& Hood 2016). Such motions can induce further shearing of the field lines along the PIL. A summary of the role of flux emergence in the formation of eruptive and non-eruptive FRs was discussed by Archontis \& Syntelis (2019).

How the FR becomes eruptive is still an open issue. Two ways have been proposed to drive its eruption. One way is through a nonideal process (e.g., magnetic reconnection) and the other is through ideal MHD instabilities (or catastrophe).

An example of magnetic reconnection that leads to an eruption is the tether-cutting mechanism. There, the magnetic field enveloping the FR reconnects through a current sheet below the FR (e.g., Moore \& Labonte 1980; Moore \& Roumeliotis 1992), commonly referred to as "flare current sheet." The upwards release of tension of the reconnected field lines at the "flare current sheet" causes fast acceleration of the FR. A variation of this process was found in Syntelis et al. (2017; hereafter, Paper I). In the latter study, the envelope magnetic field reconnects with low-lying, highly sheared field lines (J-like loops). Another example of magnetic reconnection leading to an eruption is the break-out reconnection between the envelope field and an external magnetic field. If the external field has the appropriate orientation (preferably antiparallel), it reconnects with the envelope magnetic field (e.g., Antiochos et al. 1999; Archontis \& Hood 2012; Karpen et al. 2012; 
Leake et al. 2013). This reconnection removes the downwards tension of both the envelope and the external field, causing the FR to move upwards and erupt ejectively. On the other hand, if the external field lacks the appropriate orientation (e.g., it is parallel to the envelope field), then the external field and the magnetic envelope will not reconnect. In such a configuration, an FR eruption would be suppressed by the downwards tension of both the envelope and the overlying field (confined eruption, e.g., Archontis \& Hood 2012; Leake et al. 2014).

Ideal MHD instabilities can also trigger solar eruptions. One example is the helical kink instability (Anzer 1968; Török et al. 2004). This instability occurs when the current (twist) of the FR surpasses a critical value that depends on the configuration of the FR (e.g., cylindrical, toroidal) and the line-tying effect (e.g., Hood \& Priest 1981; Török et al. 2004). Eruptions triggered by kink instability can become confined by an external magnetic field (Török \& Kliem 2005).

Another MHD instability associated with solar eruptions is the torus instability (Bateman 1978; Kliem \& Török 2006). If a toroidal current channel with major radius $R$ is placed inside an external field that drops as $B_{\text {ext }} \propto R^{-n}$, then the current channel will become unstable when $n$ exceeds a critical value, $n_{\text {crit. }}$ The torus or decay index of the field is defined as $n=$ $-\partial B_{\text {ext }} / \partial \ln R$. To calculate the decay index in simulations and observations, the usual practice is to estimate the envelope field by calculating the potential magnetic field $\left(B_{p}\right)$, and then find the decay index as $n=-z \partial \ln B_{p} / \partial z$ (e.g., Fan \& Gibson 2007; Aulanier et al. 2010). The critical value of the decay index is affected by a variety of parameters, such as the geometry of the FR, its thickness, and whether it expands during the eruption (Démoulin \& Aulanier 2010; Zuccarello et al. 2015). Numerical studies have reported values for $n_{\text {crit }}$ ranging from one to two (Fan \& Gibson 2007; Démoulin \& Aulanier 2010; Fan 2010; An \& Magara 2013; Zuccarello et al. 2015).

CMEs occur frequently and at any given day, 2-20 CMEs can be found between the Sun and a radial distance of 1 au (Lugaz et al. 2017). When a CME occurs after another one, for instance in a homologous (e.g., Liu et al. 2014; Wang et al. 2014) or sympathetic manner (e.g., Schrijver \& Title 2011; Török et al. 2011), and the second eruption is faster than the first one, the two ejecta can eventually interact, in what is called a CME-CME interaction (e.g., Gopalswamy et al. 2001). During such interaction, the CMEs' properties such as their speed, size, and expansion rates change. The nature of the collision of two CMEs is complicated, as the eruption can be inelastic, elastic, or super-elastic (e.g., Shen et al. 2017). The two colliding CMEs almost always interact through their propagating shocks (Lugaz et al. 2015). They can also interact through magnetic reconnection occurring at the interface between the two colliding FRs (e.g., Odstrcil et al. 2003; Lugaz et al. 2005, 2013; Chatterjee \& Fan 2013). The total coalescence of two CMEs is referred to as CME cannibalism (Gopalswamy et al. 2001). The full coalescence of the two colliding structures occur at different distances from the Sun, as this will depend on magnetic reconnection rates, relative speeds, field orientation, CMEs fluxes, etc. (Manchester et al. 2017).

In Paper I, we studied the onset mechanism of recurrent eruptions in the context of flux-emergence simulations. A buoyantly unstable subphotospheric horizontal flux tube formed a bipolar region that produced four recurrent eruptions.
We found that the combination of torus instability and tethercutting is the main driving mechanism for these eruptions. The kinetic energies of the eruptions were $3 \times 10^{26}-1.5 \times 10^{27} \mathrm{erg}$ and the magnetic energies were around $1 \times 10^{28} \mathrm{erg}$. Such energies correspond to small-scale eruptions. A geometrical extrapolation was performed to estimate the physical size of the erupting FRs. We found that the size of the eruptions could become comparable to the size of small CMEs. Moreover, our results showed that the second eruption occurred soon after and it was faster than the first eruption and, thus, parts of the two erupting fields collided, experiencing a partial merging. In Paper I, we could not study this merging further, as the eruptions quickly escaped the numerical domain.

In this paper, we extend our work by varying the magnitude of the magnetic field strength of the subphotospheric flux tube, $B_{0}$. The increase of $B_{0}$ leads to an increase of the magnetic pressure and the expansion of the field into the solar atmosphere, which in turn leads to an increase of the physical size of the eruptions. To follow the evolution of the eruptions in $3 \mathrm{D}$, we performed the parametric study on a larger numerical domain than the one used in Paper I. In this paper, we study the energy content and the recurrence of the eruptions. We also extend the study of the initial phase of the "collision" between two successive eruptions.

In Section 2 we describe the initial conditions of our simulations. Section 3.1 is an overview of the simulations. In Section 3.2 we discuss the energies and the frequency of the recurrent eruptions. In Section 3.3 we briefly discuss the plasma $\beta$ and Mach Alfvén speed of the erupting fields. In Section 4 we discuss the partial merging of the two first eruptions. In Section 5 we summarize and discuss our results.

\section{Numerical Setup}

We numerically solve the 3D time-dependent, resistive, compressible MHD equations in Cartesian geometry using Lare3D (Arber et al. 2001). The equations, the resistivity form, and the normalization units are shown in Paper I.

The initial condition for the simulation is a horizontal flux tube positioned at $-2.1 \mathrm{Mm}$. The axis of the flux tube is oriented along the $y$-direction, so the transverse direction is along $x$ and height is in the $z$-direction. The flux tube's magnetic field is:

$$
\begin{gathered}
B_{y}=B_{0} \exp \left(-r^{2} / R^{2}\right), \\
B_{\phi}=\alpha r B_{y},
\end{gathered}
$$

where $R=450 \mathrm{~km}$ is the tube's radius, $r$ is the radial distance from the tube axis, and $\alpha=0.4\left(0.0023 \mathrm{~km}^{-1}\right)$ is a measure of the initial twist of the subphotospheric field. For the parametric study, the magnetic field strength of the initial flux tube takes the values of $B_{0}=8(2400 \mathrm{G}), 10.5(3150 \mathrm{G}), 15(4500 \mathrm{G})$, and $20(6000 \mathrm{G})$. In each simulation, the flux tube is initially in pressure equilibrium. The flux tube is destabilized by imposing a density deficit along the axis of the flux tube, similar to Archontis et al. (2004):

$$
\Delta \rho=\frac{p_{\mathrm{t}}(r)}{p(z)} \rho(z) \exp \left(-y^{2} / \lambda^{2}\right),
$$

where $p$ is the external pressure, $p_{\mathrm{t}}$ is the total pressure within the flux tube, and $\lambda$ is the length scale of the buoyant part of the flux tube. We use $\lambda=5(0.9 \mathrm{Mm})$. 
We use a large numerical domain of a $1000^{3}$ grid with a physical size of $153^{3} \mathrm{Mm}$. We use periodic boundary conditions in the $y$ direction and open boundary conditions in the $x$ direction and at the top of the numerical domain. Closed boundary conditions are assumed at the bottom of the numerical domain.

The numerical domain consists of an adiabatically stratified subphotosheric layer at $-7.2 \mathrm{Mm} \leqslant z<0 \mathrm{Mm}$, an isothermal photospheric-chromospheric layer at $0 \mathrm{Mm} \leqslant z<1.8 \mathrm{Mm}$, a transition region at $1.8 \mathrm{Mm} \leqslant z<3.2 \mathrm{Mm}$ and an isothermal corona at $3.2 \mathrm{Mm} \leqslant z<145.8 \mathrm{Mm}$. The stratification layers and resolution are the same as those in Paper I. The initial fieldfree atmosphere is in hydrostatic equilibrium.

\section{Parametric Study: Varying the Magnetic Field Strength $B_{0}$}

\subsection{Overview}

In Paper I, we used a flux tube with $B_{0}=10.5(3150 \mathrm{G})$ inside a smaller numerical domain $\left(417^{3}\right.$ grid points). This is a relatively low magnetic field strength for $z=-2.1 \mathrm{Mm}$. The cases with $B_{0}=15,20(4500,6000 \mathrm{G})$ have field strengths that could be more indicative for magnetic fields close to the solar surface (e.g., Cheung et al. 2007). A case of lower field strength, $B_{0}=8(2400 \mathrm{G})$ was also studied.

Flux tubes with higher $B_{0}$ rise faster inside the solar interior (magnetic buoyancy is $\propto B_{0}^{2}$ ), and carry lower plasma $\beta$. Therefore, the buoyancy instability criterion, for emergence above the solar surface, is triggered earlier (Acheson 1979; Archontis et al. 2004). Above the solar surface, the flux tubes with higher $B_{0}$ expand faster due to their larger magnetic pressure and, consequently, they occupy more physical space. For all $B_{0}$ cases, we find a series of recurrent eruptions following the marked expansion of the field.

In the $B_{0}=15,20$ cases, the expansion of the field is so pronounced that the large numerical domain used is necessary in order to follow the formation of the erupting field before the overall magnetic system reaches the boundaries of the domain. In the $B=8,10.5$ cases, the expansion of the field is not so pronounced. In these cases, we use a larger numerical domain to (i) compare the energies between different $B_{0}$ and (ii) to track the erupting fields further upwards, before they exit the numerical domain.

\subsection{Energies and Recurrence}

Recurrent ejective eruptions are formed in all of the studied cases. The eruption mechanism is very similar to the one described in Paper I (Section 3; i.e., a combination of torus instability and tether-cutting reconnection).

We calculate the kinetic and magnetic energies above midphotosphere. For each $B_{0}$, we find a series of local maxima that correspond to the eruptive events. Figure 1 shows the time evolution of the kinetic and magnetic energy for $B_{0}=15$. The three kinetic energy maxima associated with eruptions are found at $t=65.7,91.4,154.3$ minutes (the first kinetic energy peak is the initial emergence above the photosphere). The time between the eruptions is $\Delta t=25.7,62.9$ minutes. For the $B_{0}=20$ we studied only the first two eruptions. The eruptions are found at $t=51.4,128.6$ minutes with a time delay of $\Delta t=77.2$ minutes. For $B_{0}=8$, the peaks of the kinetic energy (Figure 2) are found at $t=121.4,162.8,268.5$ minutes. The time delay between the eruptions is $\Delta t=41.4,105.7$ minutes.

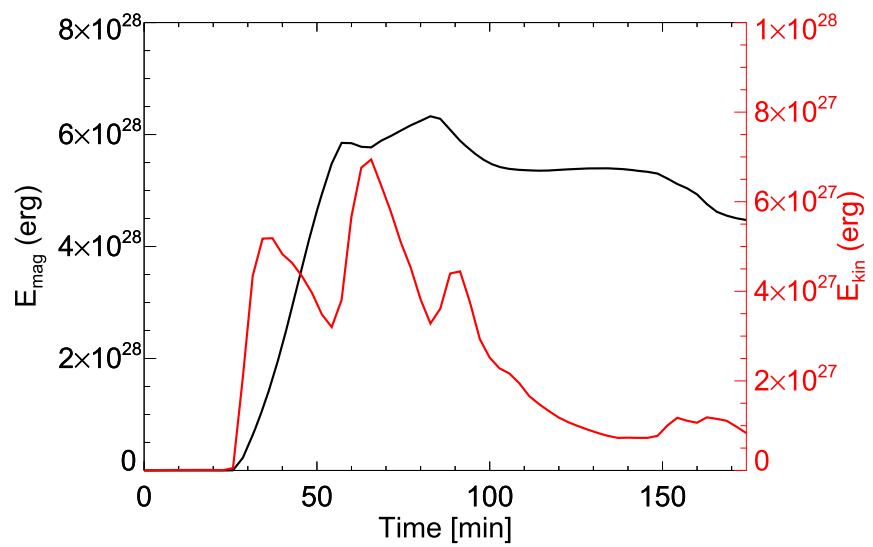

Figure 1. Magnetic (black) and kinetic (red) energy above the middle of the photospheric layer $(z=1.37 \mathrm{Mm})$ for $B_{0}=15$.

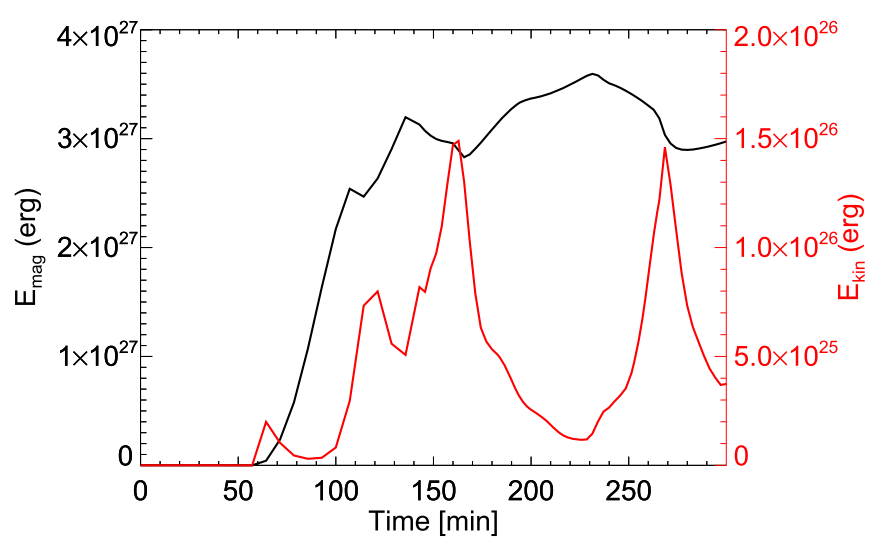

Figure 2. Magnetic (black) and kinetic (red) energy above the middle of the photospheric layer $(z=1.37 \mathrm{Mm})$ for $B_{0}=8$.

Table 1

Summary of Numerical Experiments

\begin{tabular}{lrl}
\hline \hline$B_{0}$ & \multicolumn{1}{c}{$E_{\text {mag }}$} & $E_{\text {kin }}(\mathrm{erg})$ \\
\hline 20 & $1-3 \times 10^{29}$ & $1-3 \times 10^{28}$ \\
15 & $4-6 \times 10^{28}$ & $1.5-7 \times 10^{27}$ \\
$10.5^{*}$ & $2.5-5 \times 10^{28}$ & $5 \times 10^{26}-2 \times 10^{27}$ \\
8 & $2.6-3.6 \times 10^{27}$ & $8 \times 10^{25}-1.5 \times 10^{26}$
\end{tabular}

Note. Magnitude of initial flux tube's field strength (column 1), range of magnetic and kinetic energies of eruption(s) (columns 2 and 3). The asterisk marks the simulation with the same $B_{0}$ as in Paper I.

In Paper I $\left(B_{0}=10.5\right)$, the four eruptions were found at $t=74.3, \quad 85.7, \quad 117.1, \quad 197.1 \mathrm{~s}$, with $\Delta t=11.4, \quad 31.4$, 80 minutes. These are similar to the times of the eruptions using the large domain. From the above, we find that the first eruption occurs earlier for higher $B_{0}$. However, we do not find any direct correlation between the time delay between the eruptions and $B_{0}$.

The range of the values of the kinetic and magnetic energy maxima for each $B_{0}$ is shown in Table 1 . For $B_{0}=10.5$, the magnetic energy range of the eruptions is higher than the one reported in Paper I. This is because the energies here are calculated in the large numerical domain. During and after each eruption, the large domain is filled with more magnetic field in comparison to the small domain used in Paper I. Therefore, the large domain's corona contains up to three times more magnetic energy after each eruption. 
(a)

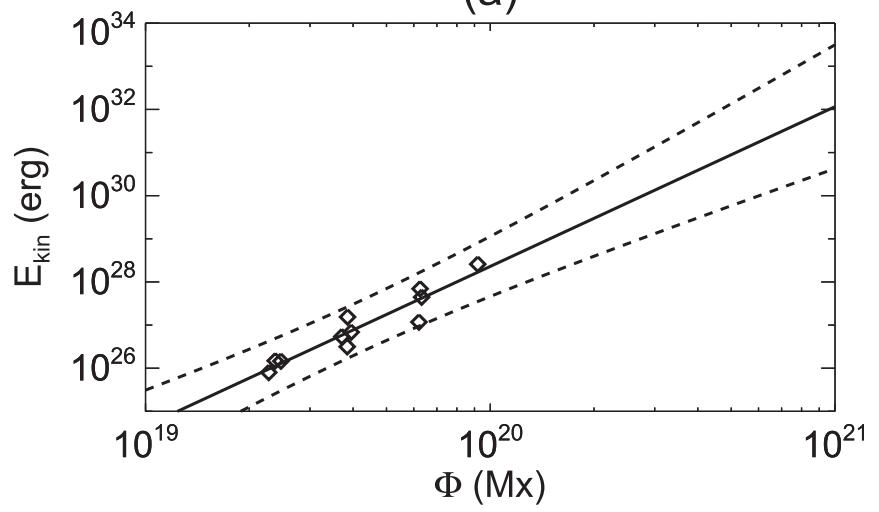

(b)

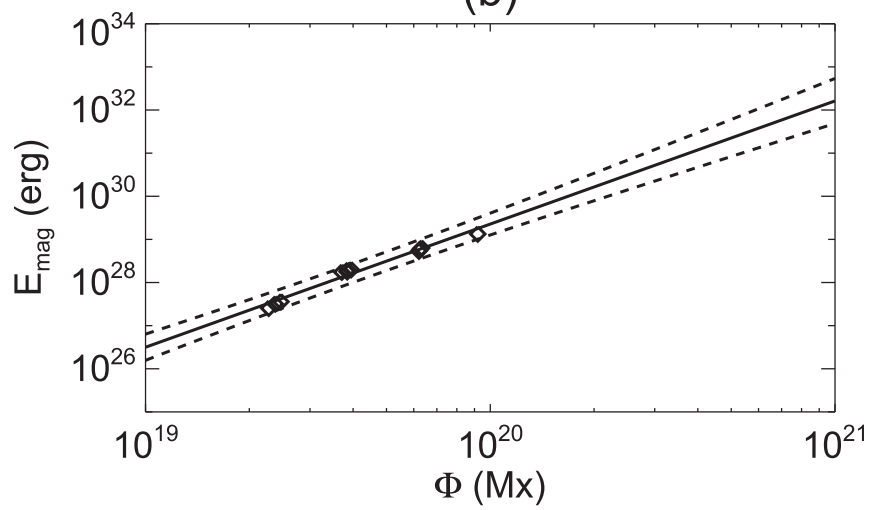

Figure 3. Kinetic (a) and magnetic (b) energy maxima over the photospheric flux at the time of the eruptions (diamonds). Energies are measured above the middle of the photospheric layer $(z=1.37 \mathrm{Mm})$. Solid lines are linear fitted lines. Dashed lines are the $95 \%$ confidence level of the fitted lines.

Next, for all of our eruptions, we take the values of the kinetic energy peaks and the values of the preceding magnetic energy peaks and plot them as a function of the photospheric flux at that time (diamonds in Figures 3(a) and (b)). We perform a linear regression to assess any potential linear scaling. In panel (a), the fitted line (solid line) is $\log E_{\text {kin }}=$ $(3.7 \pm 0.4) \log \Phi+(-45.7 \pm 8.2)$ with an $r^{2}=0.90$, and in panel (b) is $\log E_{\text {mag }}=(2.8 \pm 0.4) \log \Phi+(-27.7 \pm 3.4)$ with an $r^{2}=0.98$. The $95 \%$ confidence interval of our fitted lines is shown with dashed lines. The total magnetic field energy above the photosphere correlates well with the photospheric flux. The kinetic energy associated with the eruptions is more spread as the same emerging region can produce eruptions of different kinetic energies. As our statistical sample is relatively small $(n=11)$, the confidence interval is relatively wide for larger values of the photospheric fluxes. However, based on our extrapolation, for a typical AR flux of $10^{21} \mathrm{Mx}$, we predict energies of $4 \times 10^{30}-3 \times 10^{33} \mathrm{erg}$, which are typical energies of CMEs.

\subsection{Plasma $\beta$ and Alfvén Speed}

Figure 4 shows the temperature (first column) and density (second column) at the $x z$-midplane of the first two eruptions of the $B=10.5$ case. In panel (a1), the magnetized plasma between $z \approx 35-85 \mathrm{Mm}$ belongs to the the first eruption. The FR center is located inside its central region $(z=60 \mathrm{Mm}, \mathrm{FR} 1$ asterisk). Below $z \approx 30 \mathrm{Mm}$ we find the second erupting structure (the FR2 asterisk marks the center of the second FR).
At that time, the second FR has just entered the fast-rise phase during its eruption. Panel (a2) shows a later time when both FRs undergo a full ejective eruptive phase. Both erupting structures have a hot and dense central region (panels (b1) and (b2)), which is surrounded by a hot and dense outermost region (similar to Paper I).

In panels (c1) and (c2) we plot the plasma beta, $\beta=P_{g} / P_{m}$, where $P_{g}$ and $P_{m}$ are the gas and magnetic pressure respectively. Both eruptions have $\beta<1$ everywhere and therefore the eruptions are magnetically dominated. One exception is the "flanks" of the erupting structures (i.e., the interface between the magnetized eruptions and the nonmagnetized atmosphere, for e.g., around $x= \pm 45 \mathrm{Mm}$ ), where $\beta>1$ (red and white color). We find that the aerodynamical drag deforms these high beta regions (e.g., around $x=$ $\pm 45 \mathrm{Mm}$ and $z \in[60,100] \mathrm{Mm})$.

Another region where $\beta$ can be around unity is the flare current sheet. Panel (c2) shows as an example the flare current sheet of the second eruption (white color around $x \in[-5,5]$ and $z \in[5,40])$. There, plasma $\beta$ increases locally due to both the increase of the plasma density and temperature and due to the lower magnetic pressure inside the current sheet. It has been reported that during fast reconnection high- $\beta$ plasmoids can result from the fragmentation of the current sheet (e.g., Karlický et al. 2012).

In panels (d1) and (d2) we show the Mach Alfvén number of the two eruptions $\left(M_{a}=v / v_{a}\right)$, where $v$ is the magnitude of the velocity field and $v_{a}=B / \sqrt{4 \pi \rho}$ is the Alfvén speed). Inside the erupting structures and around the center of the FR, we find regions of high $M_{a}$ (purple regions). The high $M_{a}$ indicates shocks inside the erupting structures. These shocks are formed during the eruption of each of the FRs. These shocks will be further discussed in Section 4.

The $B_{0}=15,20$ cases are similar to the $B_{0}=10.5$ one. For lower the magnetic field strength case, $B_{0}=8$, we show the first eruption (Figure 5). Here, we do not find regions of $M_{a}>1$ inside the erupting structure, besides inside the flare current sheet. We still find $\beta \geqslant 1$ regions around the "flanks" of the erupting field.

\section{Partial Merging of the Erupting Fields}

In Paper I, we studied the height-time and velocity profiles of the eruptions of the $B_{0}=10.5$ case. The second erupting FR was faster than the first. Before the second erupting structure exited the numerical domain, it encountered the magnetic field of the first one. This caused a deceleration of the second FR (see second FR's velocity profile, black line, Figure 9(a), Paper I). This is what we refer to as "collision" of the two erupting magnetic structures. In this paper, we follow this "collision" further, by following the eruption further upwards in the large numerical domain.

Figure 4(a1) shows the temperature of the two erupting FRs. Between the two erupting structures, (around $z \approx 33 \mathrm{Mm}$ and $x=0 \mathrm{Mm}$ ), there is a thin interface with hot plasma. This is part of the flare current sheet underneath the first eruption. In Figure 4(a2), when both FR1 and FR2 undergo a full ejective eruptive phase, the first erupting structure is found between $z \approx 80-130 \mathrm{Mm}$ and the second below $z \approx 80 \mathrm{Mm}$. The two structures "collide" at around $z \approx 80 \mathrm{Mm}$.

Notice that between panel (a1) and panel (a2), the width of leading eruption (its extent along $z$ decreases. This "contraction" of the leading eruption is commonly found during 


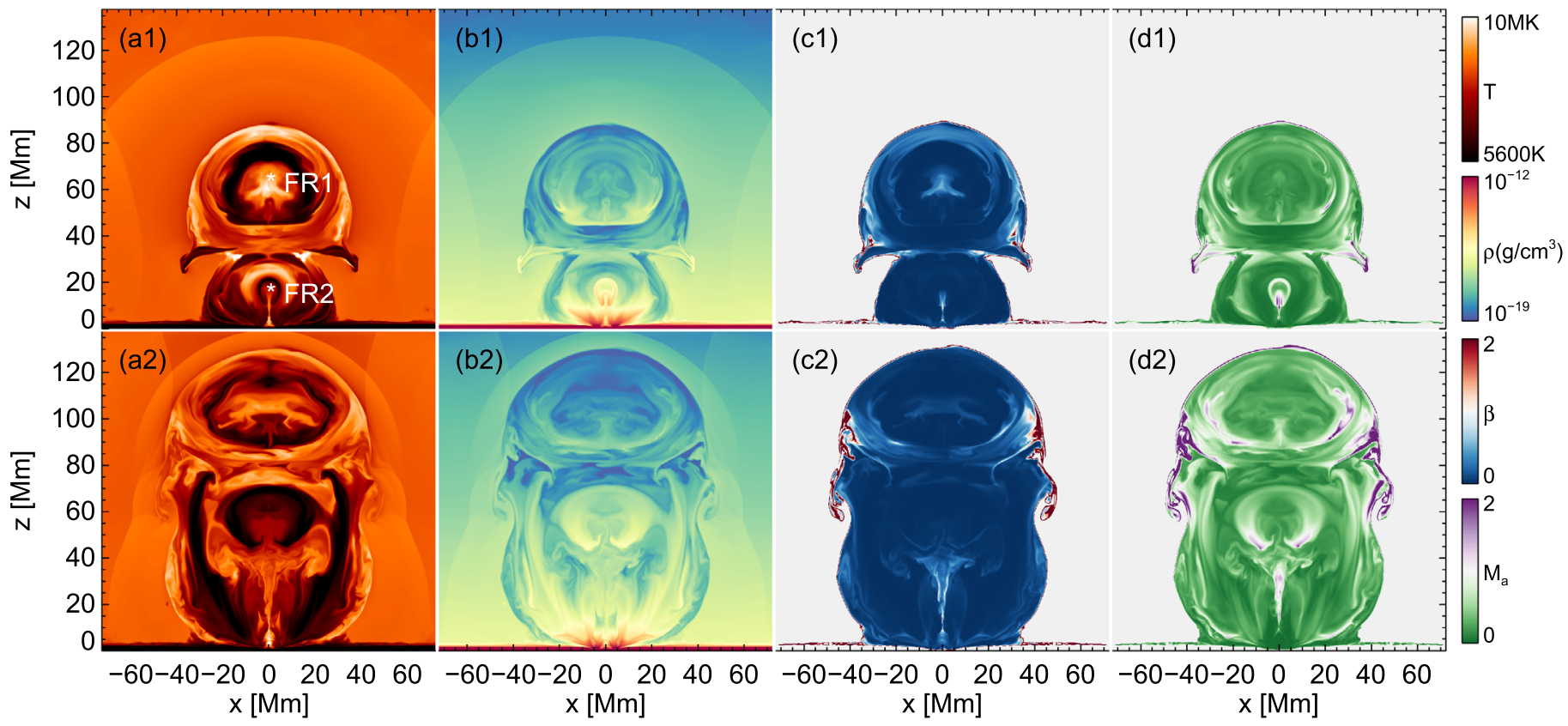

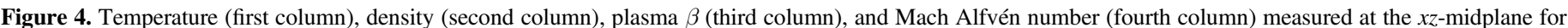

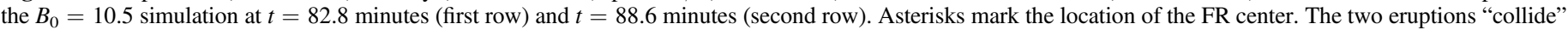
between the two time instances. Gray background in the third and fourth columns show the nonmagnetized background.

CME-CME interactions and it is due to the compression of the leading structure during the "collission" (e.g., Manchester et al. 2017). Also, during the "collision" of the two erupting structures, the high $M_{a}$ regions inside the first eruption (panel (d1)) become more enhanced and extended (panel (d2)). This demonstrates that the compression between the colliding structures can form/enhance shocks inside two colliding eruptions. Such shocks inside CMEs have been reported to be formed/enhanced during the collision of CMEs/ICMEs (e.g., Lugaz et al. 2015, 2017).

To understand better the structure of the erupting field(s), we use visualization of selected field lines, which are traced from various locations within the overall erupting system. In Figure 6(a), we trace field lines from the FR1 center in yellow (as in Figure 7(c) of Paper I). With red color, we trace field lines from an area that surrounds the central region of the erupting field (i.e., the hot circular cross-section area, located around $-40 \mathrm{Mm}<x<40 \mathrm{Mm}$ and $85 \mathrm{Mm}<z<125 \mathrm{Mm}$ in Figure 4(a2)). These field lines had undergone EnvelopeEnvelope tether-cutting reconnection, as discussed in Paper I (and are similar to the red field lines in Figure 7(c) of Paper I). The field lines, which go through the center of the FR2 are shown in orange color. The erupting field that envelopes FR2 is plotted with green lines. These lines are traced from the high temperature region around $z \approx 75 \mathrm{Mm}$ in Figure 4(a2). The orange and green field lines correspond to the FR and envelope field in Figure 8(f) of Paper I (with yellow and green color, respectively).

Studying the topology of the field lines, we notice that the two initially separated structures start to partially merge after they "collide." This partial merging happens through magnetic reconnection of their fieldines, which occurs mainly at two different sites. The first reconnection site is shown in Figure 6(b) (this is the regions around $z \approx 80 \mathrm{Mm}$, Figure 4, (a2)). A current sheet (purple isosurface) is formed between the green and the red field lines. These sets of field lines reconnect with each other at this current sheet. This reconnection forms field lines that start from the one end of the green envelope field lines, twist around FR1, and end at the footpoints of the red field lines (blue line, Figure 6(c)). The reconnection also forms a field line that starts on one end of the green envelope field lines and ends at the footpoints of the red field lines (orange line). This new line has less downwards magnetic tension than the green line of Figure 6(b). Therefore, after the reconnection it is possible that the upwards rise of FR2 will be further assisted.

The second reconnection site is shown in Figure 6(d) (purple isosurface). This is the location of the flare current sheet of the second eruption. As FR1 (yellow lines) moves upwards, its footpoints move toward the vicinity of the flare current sheet of the second eruption. There, the yellow FR1 field lines reconnect with other FR1 field lines via tether-cutting (i.e., the envelope-envelope tether-cutting mechanism discussed in Paper I). This reconnection forms twisted field lines (e.g., light blue line, Figure 6(e)) and adds flux to the low-lying postreconnection arcade (e.g., gray line). Notice that the light blue line twists around both FR1 and FR2, linking the two structures (Figure 6(f)). Furthermore, the upwards tension release of the field lines similar to the light blue field line push will push FR2 upwards, bringing FR1 and FR2 closer together. This reconnection can occur many times. For instance, a field line similar to the light blue one of panel (e) can reconnect with lines similar to the yellow lines of panel (d), forming field lines with high twist.

Because the system is very complex, it is very likely that there are more reconnection sites within the overall erupting volume of the field. However, the blue and light blue field lines are the two main sets of new field lines found after the "collision" of the two magnetic structures. For simplicity, we refer to the linkage of the two erupting field structures, through the blue and light blue lines, as partial merging. 

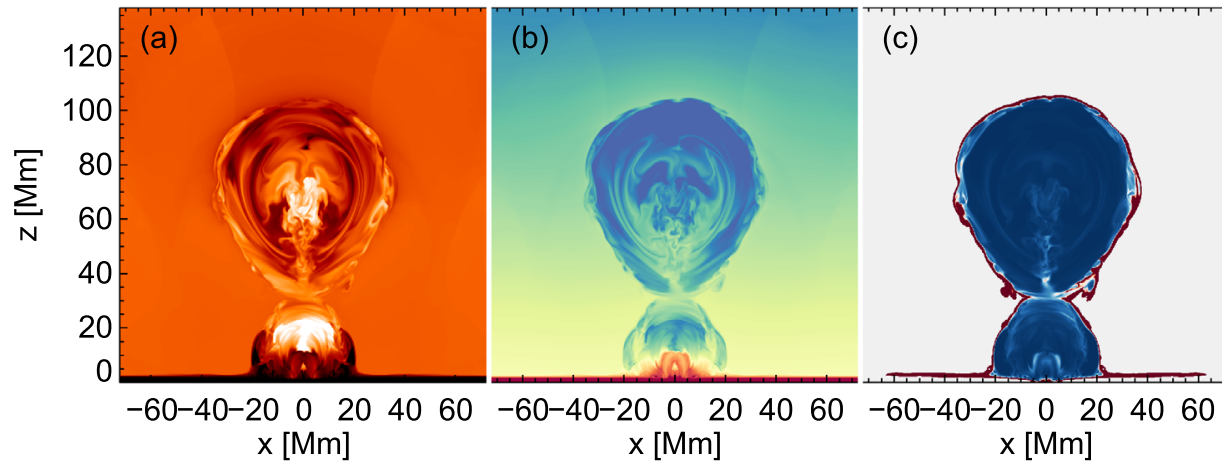

(d)

Figure 5. Temperature (a), density (b), plasma $\beta$ (c), and Mach Alfvén number (d) measured at the $x z$-midplane for the first eruption of the $B_{0}=8$ simulation at $t=128.6$ minutes. The gray background in panels (c) and (d) show the nonmagnetized background.

We were unable to study the partial merging in the other $B_{0}$ cases. The reason for that is that in all other cases, the time interval between the onset of two successive eruptions is larger than the one found in the $B_{0}=10.5$ case. Therefore, even if the second eruption was faster than the first eruption, the first eruption would have partially or fully exited the numerical domain before the second erupting structure "collided" with it.

\section{Summary and Discussion}

In this work, we performed a parametric study on the magnetic field strength $\left(B_{0}\right)$ of a subphotospheric magnetic flux tube. We focused on the evolution of the eruptions, which occurred after the emergence of the magnetic field at the solar surface and the formation of a small AR. In this study, we used a relatively large numerical domain $\left(1000^{3}\right.$ grid points, $145.6^{3}$ $\mathrm{Mm})$. The values of $B_{0}$ used are shown in Table 1 . In all the $B_{0}$ cases, we found that recurrent eruptions were triggered above the PIL of the ARs. The $B_{0}=8,10.5$ cases $\left(B_{0}=10.5\right.$ is also the Paper I case) yielded kinetic energies of $8 \times$ $10^{25}-2 \times 10^{27}$ erg. Such energies correspond to small-scale eruptive events. For $B_{0}=15,20$, the kinetic energy of the eruptions ranged from $1 \times 10^{27}-3 \times 10^{28} \mathrm{erg}$, which is comparable to the kinetic energy of small-scale CMEs (Vourlidas et al. 2010). A further increase of $B_{0}$ (e.g., up to $\left.B_{0}=35\right)$ might produce energies of the order of an average CME (around $10^{29} \mathrm{erg}$ ). Such work would require even larger numerical domains, which are computationally very expensive. Also, a spherical grid would be required to study in detail the eruptions of that scale.

In all of our cases, the first eruption is formed earlier in time for higher $B_{0}$. For $B_{0}=8$, the frequency of the eruptions is lower compared to the $B \geqslant 10.5$ and above. For higher $B_{0}$, we do not find a clear correlation between the frequency of the eruptions and the increase of the initial subphotospheric magnetic field strength. Therefore, we cannot associate the frequency of the eruptions with $B_{0}$.

For all of our eruptions (11 in total) we plotted their kinetic energy and the atmospheric magnetic energy prior to the eruption as a function of the photospheric flux. We found that both quantities follow linear scaling in a log-log plot. Our sample ranges across a relatively small range of photospheric fluxes, and the resulting energies of the eruptions are comparable to small-scale prominence eruptions (mini or micro CMEs, e.g., Innes et al. 2009; Raouafi et al. 2010; Hong et al. 2011) and small CMEs (e.g., Vourlidas et al. 2010; Reeves et al. 2015). Similar energies are reported in simulations of small-scale eruption such as the solar jets (e.g., Liu et al. 2016; Raouafi et al. 2016). The extrapolation of our linear fit to AR fluxes $\left(10^{21} \mathrm{Mx}\right)$ results to typical kinetic energies for large scale CMEs $\left(10^{30}-10^{33} \mathrm{erg}\right)$. If such a scaling is indeed occurring in solar eruptions, it would connect eruptions of both smaller and larger scales. It would be interesting to study whether such a linear scaling remained after including events with typical energies of small and large CMEs and events with typical energies of jets (both standard and blowout ones). Such a scaling would be a strong indication on the jet-CME connection (e.g., Wyper et al. 2017).

We also studied the distribution of plasma $\beta$ inside the erupting structures. We found that indeed the eruptions are magnetically driven because $\beta<1$ inside the erupting field. Plasma $\beta$ becomes greater than one at the outermost region of the magnetized structures (the "flanks" of the eruptions). This high beta region can be deformed by the aerodynamical drag.

In Paper I, we studied in detail the $B_{0}=10.5$ case using a small numerical domain $\left(417^{3}\right.$ grid points, $\left.64.8^{3} \mathrm{Mm}\right)$. There, the second eruption was found to be faster than the first one, with signs of "collision" between the two successive eruptions. In this work, using the larger numerical domain, we were able to follow this "collision" further upwards. We found that it leads to the partial merging of the two magnetic structures. The merging occurred by the reconnection of field lines, at two (at least) sites. The first reconnection site was located at the interface between the two colliding structures adding twist around the first erupting FR while removing flux from the following erupting FR. The second reconnection site was the flare current sheet underneath the second erupting FR. There, the field lines of the leading FR reconnected through tethercutting, forming lines that surrounding both FRs, magnetically linking the two structures.

Simulations studying CME-CME interaction of two erupting FRs propagating through the solar wind (e.g., Odstrcil et al. 2003; Lugaz et al. 2005, 2013), show that reconnection occurs always at the interface between the first and second eruption. Depending on the relative orientation of the FR's axis, this process can lead to the full or not merging of the two structures (Lugaz et al. 2013). Here, we show that the tether-cutting reconnection of the leading FR's field lines can magnetically connect the two erupting structures with an additional way that has not been reported before. We cannot conclude whether the two FRs of our simulation would fully merge and become cospatial (as in the study of Chatterjee \& Fan 2013). It is likely that our experiment describes aspects of the initial phase of a cannibalistic process. 


$$
\frac{1}{2} \frac{1}{2}
$$


During the collision of the two structures, regions of high Alfvén Mach number develop. We interpret these structures as shocks inside the colliding FRs. Observations of CME-CME interactions in the interplanetary medium have reported the development of shocks inside the colliding CMEs (e.g., Lugaz et al. 2015, 2017; Shen et al. 2017).

The authors would like to thank the referee for constructive comments. This project has received funding from the Science and Technology Facilities Council (UK) through the consolidated grant ST/N000609/1. The authors acknowledge support by the Royal Society. This work was supported by computational time granted from the Greek Research \& Technology Network (GRNET) in the National HPC facility-ARIS.

\section{ORCID iDs}

P. Syntelis (ib https://orcid.org/0000-0002-6377-0243

K. Tsinganos (1D https://orcid.org/0000-0002-6017-7399

\section{References}

Acheson, D. J. 1979, SoPh, 62, 23

An, J. M., \& Magara, T. 2013, ApJ, 773, 21

Antiochos, S. K., DeVore, C. R., \& Klimchuk, J. A. 1999, ApJ, 510, 485

Anzer, U. 1968, SoPh, 3, 298

Arber, T., Longbottom, A., Gerrard, C., \& Milne, A. 2001, JCoPh, 171, 151

Archontis, V., \& Hood, A. W. 2012, A\&A, 537, A62

Archontis, V., Moreno-Insertis, F., Galsgaard, K., Hood, A., \& O'Shea, E. 2004, A\&A, 426, 1047

Archontis, V., \& Syntelis, P. 2019, arXiv:1904.06274

Archontis, V., \& Török, T. 2008, A\&A, 492, L35

Aulanier, G., Török, T., Démoulin, P., \& DeLuca, E. E. 2010, ApJ, 708, 314

Bateman, G. 1978, MHD Instabilities (Cambridge, MA: MIT Press)

Canou, A., \& Amari, T. 2010, ApJ, 715, 1566

Chatterjee, P., \& Fan, Y. 2013, ApJL, 778, L8

Cheng, X., Zhang, J., Liu, Y., \& Ding, M. D. 2011, ApJL, 732, L25

Cheung, M. C. M., Schüssler, M., \& Moreno-Insertis, F. 2007, A\&A, 467, 703

Chintzoglou, G., Patsourakos, S., \& Vourlidas, A. 2015, ApJ, 809, 34

Démoulin, P., \& Aulanier, G. 2010, ApJ, 718, 1388

DeVore, C. R., \& Antiochos, S. K. 2008, ApJ, 680, 740

Fan, Y. 2001, ApJL, 554, L111

Fan, Y. 2009, ApJ, 697, 1529

Fan, Y. 2010, ApJ, 719, 728

Fan, Y., \& Gibson, S. E. 2007, ApJ, 668, 1232

Gopalswamy, N., Yashiro, S., Kaiser, M. L., Howard, R. A., \& Bougeret, J. L. 2001, ApJL, 548, L91

Green, L. M., \& Kliem, B. 2009, ApJL, 700, L83

Green, L. M., Kliem, B., \& Wallace, A. J. 2011, A\&A, 526, A2

Hong, J., Jiang, Y., Zheng, R., et al. 2011, ApJL, 738, L20

Hood, A. W., \& Priest, E. R. 1981, GApFD, 17, 297

Innes, D. E., Genetelli, A., Attie, R., \& Potts, H. E. 2009, A\&A, 495, 319

Karlický, M., Bárta, M., \& Nickeler, D. 2012, A\&A, 541, A86
Karpen, J. T., Antiochos, S. K., \& DeVore, C. R. 2012, ApJ, 760, 81 Kliem, B., \& Török, T. 2006, PhRvL, 96, 255002

Leake, J. E., Linton, M. G., \& Antiochos, S. K. 2014, ApJ, 787, 46

Leake, J. E., Linton, M. G., \& Török, T. 2013, ApJ, 778, 99

Liu, J., Wang, Y., Erdélyi, R., et al. 2016, ApJ, 833, 150

Liu, Y. D., Luhmann, J. G., Kajdič, P., et al. 2014, NatCo, 5, 3481

Lugaz, N., Farrugia, C. J., Manchester, W. B. I., \& Schwadron, N. 2013, ApJ, 778,20

Lugaz, N., Farrugia, C. J., Smith, C. W., \& Paulson, K. 2015, JGRA, 120, 2409

Lugaz, N., Manchester, W. B. I., \& Gombosi, T. I. 2005, ApJ, 634, 651

Lugaz, N., Temmer, M., Wang, Y., \& Farrugia, C. J. 2017, SoPh, 292, 64

Lynch, B. J., Antiochos, S. K., DeVore, C. R., Luhmann, J. G., \& Zurbuchen, T. H. 2008, ApJ, 683, 1192

Mackay, D. H., \& van Ballegooijen, A. A. 2006, ApJ, 641, 577

Magara, T., \& Longcope, D. W. 2001, ApJL, 559, L55

Manchester, W., Kilpua, E. K. J., Liu, Y. D., et al. 2017, SSRv, 212, 1159

Manchester, W. B., Vourlidas, A., Tóth, G., et al. 2008, ApJ, 684, 1448

Manchester, W., IV 2001, ApJ, 547, 503

Manchester, W., IV, Gombosi, T., DeZeeuw, D., \& Fan, Y. 2004, ApJ, 610,588

Moore, R. L., \& Labonte, B. J. 1980, in IAU Symp. 91, Solar and Interplanetary Dynamics, ed. M. Dryer \& E. Tandberg-Hanssen (Dordrecht: Reidel), 207

Moore, R. L., \& Roumeliotis, G. 1992, in IAU Coll. 133: Eruptive Solar Flares 399, Lecture Notes in Physics, ed. Z. Svestka, B. V. Jackson, \& M. E. Machado (Berlin: Springer), 69

Nitta, N. V., \& Hudson, H. S. 2001, GeoRL, 28, 3801

Odstrcil, D., Vandas, M., Pizzo, V. J., \& MacNeice, P. 2003, in AIP Conf. Ser. 679, Solar Wind Ten, ed. M. Velli et al. (Melville, NY: AIP), 699

Parker, E. N. 1955, ApJ, 121, 491

Patsourakos, S., Georgoulis, M. K., Vourlidas, A., et al. 2016, ApJ, 817, 14

Patsourakos, S., Vourlidas, A., \& Stenborg, G. 2013, ApJ, 764, 125

Raouafi, N.-E., Georgoulis, M. K., Rust, D. M., \& Bernasconi, P. N. 2010, ApJ, 718, 981

Raouafi, N. E., Patsourakos, S., Pariat, E., et al. 2016, SSRv, 201, 1

Reeves, K. K., McCauley, P. I., \& Tian, H. 2015, ApJ, 807, 7

Schrijver, C. J., \& Title, A. M. 2011, JGRA, 116, A04108

Shen, F., Wang, Y., Shen, C., \& Feng, X. 2017, SoPh, 292, 104

Sturrock, Z., \& Hood, A. W. 2016, A\&A, 593, A63

Sturrock, Z., Hood, A. W., Archontis, V., \& McNeill, C. M. 2015, A\&A, 582, A76

Syntelis, P., Archontis, V., \& Tsinganos, K. 2017, ApJ, 850, 95

Syntelis, P., Gontikakis, C., Patsourakos, S., \& Tsinganos, K. 2016, A\&A, 588, A16

Török, T., \& Kliem, B. 2005, ApJL, 630, L97

Török, T., Kliem, B., \& Titov, V. S. 2004, A\&A, 413, L27

Török, T., Panasenco, O., Titov, V. S., et al. 2011, ApJL, 739, L63

van Ballegooijen, A. A., \& Martens, P. C. H. 1989, ApJ, 343, 971

Vourlidas, A., Howard, R. A., Esfandiari, E., et al. 2010, ApJ, 722, 1522

Vourlidas, A., Lynch, B. J., Howard, R. A., \& Li, Y. 2013, SoPh, 284, 179

Vourlidas, A., Syntelis, P., \& Tsinganos, K. 2012, SoPh, 280, 509

Wang, R., Liu, Y. D., Yang, Z., \& Hu, H. 2014, ApJ, 791, 84

Wang, Y., Liu, L., Shen, C., et al. 2013, ApJL, 763, L43

Wyper, P. F., Antiochos, S. K., \& DeVore, C. R. 2017, Natur, 544, 452

Yardley, S. L., Green, L. M., van Driel-Gesztelyi, L., Williams, D. R., \& Mackay, D. H. 2018, ApJ, 866, 8

Zuccarello, F. P., Aulanier, G., \& Gilchrist, S. A. 2015, ApJ, 814, 126

Zuccarello, F. P., Seaton, D. B., Mierla, M., et al. 2014, ApJ, 785, 88 